\title{
A Typical Presentation of Madelung Disease
}

\author{
Maisel Lotan Adi*, Retchkiman Meir and Gronovich Yoav \\ Department of Plastic \& Reconstructive Surgery, Shaare Zedek Medical Center, Jerusalem, Israel
}

\begin{abstract}
We present a unique case of a sixty two year old woman with symmetric lipomatosis of the lower back, similar to cup C female breast. Lipoma is the most common type of soft tissue mesenchymal tumor, commonly found in the upper back, neck, shoulders and abdomen, and rarely in the face, hands or feet. When lipomatosis is symmetrical it is often termed Multiple Symmetrical Lipomatosis (MSL), also known as Madelung disease, a rare syndrome of unknown etiology. Our patient was referred with large symmetrical subcutaneous masses in her lower back similar to cup $C$ female breast. Prior to her surgery, the patient was referred to ultrasonography of the lower back which demonstrated two large subcutaneous lesions with an average size of $12.5 \times 3 \times 14 \mathrm{~cm}$. Lesions were excised under general anesthesia, with the left lipoma weighing $535 \mathrm{gr}$ ', and the right $425 \mathrm{gr}$, and sent for pathological examination. Surgical drains were removed two days after the procedure, and the patient was discharged. Pathology confirmed our clinical diagnosis, and 18 months after the procedure-the surgical wounds healed to the patient's satisfaction.
\end{abstract}

We believe that although this disease remains rare, the deferential diagnosis between simple lipoma, MSL and obesity may contribute to current underdiagnoses of MSL. It is important to accurately diagnose MSL as it may be related to systemic comorbidities.

Plastic surgeons should bear in mind that clinical presentation may vary, as for our patient, and must be familiar with this disease and its management.

Keywords: Madelung disease; Symmetric lipomatosis; Lipoma

\section{Introduction}

Lipoma, a benign mesenchimal lesion, is the most common type of soft tissue tumor. It is usually located superficially in the subcutaneous tissue. Less frequently it can be located deeper, under the fascia or within the muscles [1]. Lipoma is mostly asymptomatic but may compress near structures, depending on its size and location. Lipoma is most commonly found in the upper back, neck, shoulder or abdomen, but can be found anywhere in the body [2]. When lipomatosis is symmetrical it is often termed Multiple Symmetrical Lipomatosis (MSL), also known as: Madelung Disease, Benign Symmetrical Lipomatosis, Launois-Bensaude Adenolipomatosis, Brodie Syndrome or Buschke Disease. MSL is characterized by the presence of large adipose masses, minimally encapsulated, soft, painless, slowly growing and classically located at the cervical or limb area. The disease generally occurs in men aged 30 to 60 , women/men ratio ranges between $1 / 15-1 / 30$, and is seen mostly in mediterranean countries [3]. MSL is associated with increased alcohol intake in up to $95 \%$ of cases and patients have also been known to suffer from other comorbidities such as peripheral neuropathy (80\% of patients), macrocytic anemia, alcoholic fatty liver or cirrhosis, hyperuricemia, hyperproteinemia, high lipid blood levels, arterial hypertension, COPD, obesity and diabetes mellitus. Differential diagnosis with cushing disease, simple obesity, neck cysts, salivary and thyroid gland disease, leukemia and soft tissue sarcoma. The etiology of MSL remains unknown. Although there might be a genetic predisposition, most cases are sporadic with exact risk factors and pathophysiology unknown [4]. As MSL is highly associated with alcohol intake, theories suggests that alcohol may influence enzymatic process in the mitochondria or impair adrenergic lipolysis and lead to uncontrolled emergence of fat deposits in the body [5-7]. Other theories include increased lipoprotein lipase activity, mitochondrial DNA mutations or sympathetic denervation of brown fat adipocytes leading to hypertrophy [4]. Two variants are described by the most widely used Enzi classification: Type I MSL where lipomatosis may be profound and is distributed mostly around the neck (nuchal, subclavian and deltoid regions) forming the "Madelung collar", and type II where lipomatosis is located more caudally, without affecting the neck. In type II MSL patients, lipomatosis is not profound and they lack lipodystrophy of the arms and legs [6]. Diagnosis of MSL is clinical, based on symmetrical distribution of fatty masses [3]. Imaging may assist diagnosis: chest radiographs may show abnormal symmetrical accumulation, ultrasound and CT may evaluate disease extent; however MRI is the best diagnostic tool for evaluating the spread of adipose tissue, presence of tracheal compression and vascular topography within the fat mass. Patients initially complain of aesthetic changes but with long standing lipomas, reduced neck mobility or compression of respiratory structures may appear $[8,9]$. Indications for treatment include breathing difficulties, dysphagia, decreased head movements and aesthetic reasons. Possible treatment options for patients with aesthetic or functional deformities include surgical excision and liposuction. Surgical excision remains the most common and effective treatment modality, however recurrence may occur for all treatment modalities. Possible surgical complications are hematoma, seroma, recurrence or neuropraxia. Dietary treatment is ineffective as lipomas remain even with cachexia $[4,7,10,11]$.

\section{Case Report}

A sixty two year old healthy Caucasian female journalist, married with two children, was referred to our department with large symmetrical subcutaneous masses in her lower back, similar to cup C

*Corresponding author: Maisel Lotan Adi, MD, Department of Plastic \& Reconstructive Surgery, Shaare Zedek Medical Center, Jerusalem, Israel, Tel: 009722666375; E-mail: lotan.adi@gmail.com

Received February 06, 2016; Accepted April 04, 2016; Published April 13, 2016

Citation: Adi ML, Meir R, Yoav G (2016) A Typical Presentation of Madelung Disease. J Gen Pract 4: 240. doi: 10.4172/2329-9126.1000240

Copyright: ( 2016 Adi ML, et al. This is an open-access article distributed under the terms of the Creative Commons Attribution License, which permits unrestricted use, distribution, and reproduction in any medium, provided the original author and source are credited. 
female breast (Figure 1). The patient has no permanent medications, doesn't smoke or drink alcohol. She has no known allergies or family history of lipomatosis. Lesions were asymptomatic and grew slowly over a period of twenty years, but recently growth was accelerated. Physical examination showed two soft symmetrical subcutaneous masses in the lumbar area, on each side of the vertebral spine. Ultrasonography of the lower back was performed preoperatively, and revealed subcutaneous lesions with an average size of $12.5 \times 3 \times 14 \mathrm{~cm}$ consistent with lipoma. Lesions were excised under general anesthesia, with the left lipoma weighing $535 \mathrm{~g}$ and the right $425 \mathrm{~g}$ (Figure 2), and sent for pathological examination. After lipoma removal, skin reduction and wound closure were first addressed with periareolar skin pattern reduction and purse-string suture (Figure 3). Because of increased tension closure, a mastectomy horizontal scar was made. Surgical drains were removed after two days and the patient was discharged. Pathology report confirmed clinical diagnosis of lipoma and upon eighteen months of follow up, surgical wounds have healed to the patient's satisfaction (Figure 4). There were no complications nor evidence of recurrence up to 18 months follow up.

\section{Discussion}

MSL is a rare syndrome of unknown etiology characterized by indolent growth of symmetrical asymptomatic fatty masses. The disease mostly occurs in white males of Mediterranean origin, in their fourth decade [8]. Unlike lipomas, these lesions lack a distinct membranous capsule. Although this disease remains rare, the deferential diagnosis between simple lipoma, MSL and obesity may contribute to current underdiagnosis of MSL. Moreover, they are often considered as simple adipose tissue due to obesity [3,9]. Other possible reasons for underdiagnosis are the lack of strict inclusion criteria as to the localization and dimensions of lipomas in MSL due to scarce

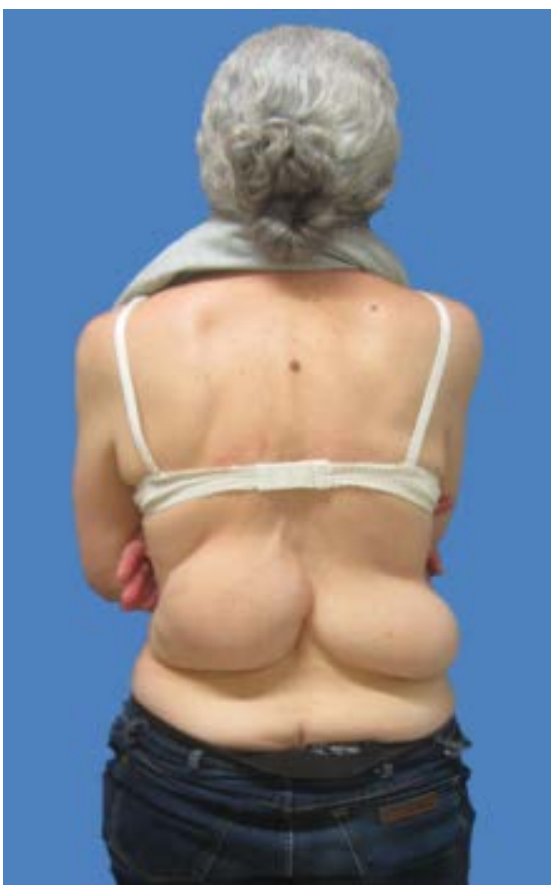

Figure 1: Clinical appearance before surgery with bilateral symmetric lipomatosis of lower back.

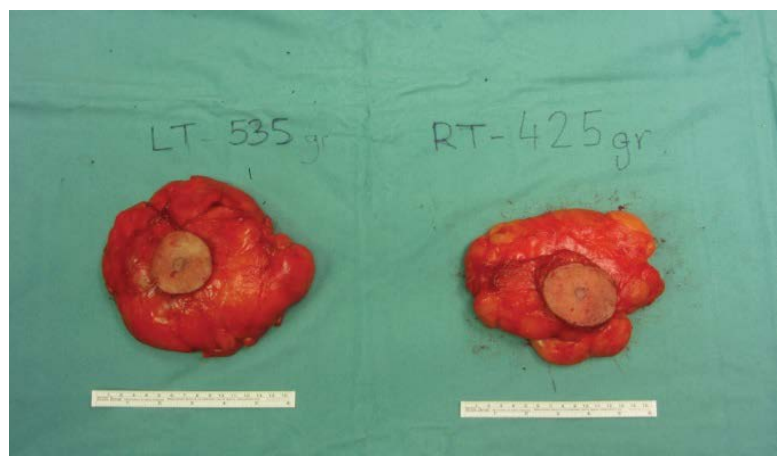

Figure 2: Complete surgical removal of the lesions with skin excess. Notice that lipomas lack a distinct membranous capsule.

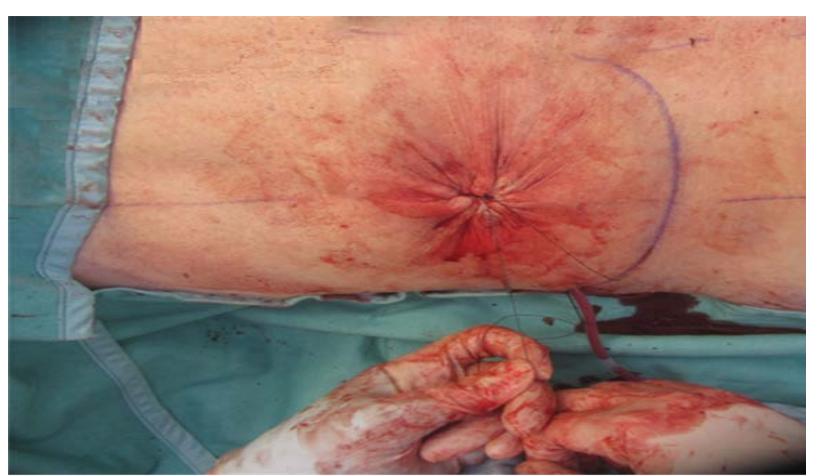

Figure 3: After lipoma removal, skin reduction and wound closure were first addressed with a "Periareolar skin pattern reduction" and pursestring suture. However, due to high tension closure, a "mastectomy type" horizontal scar was made that better addressed skin excess.

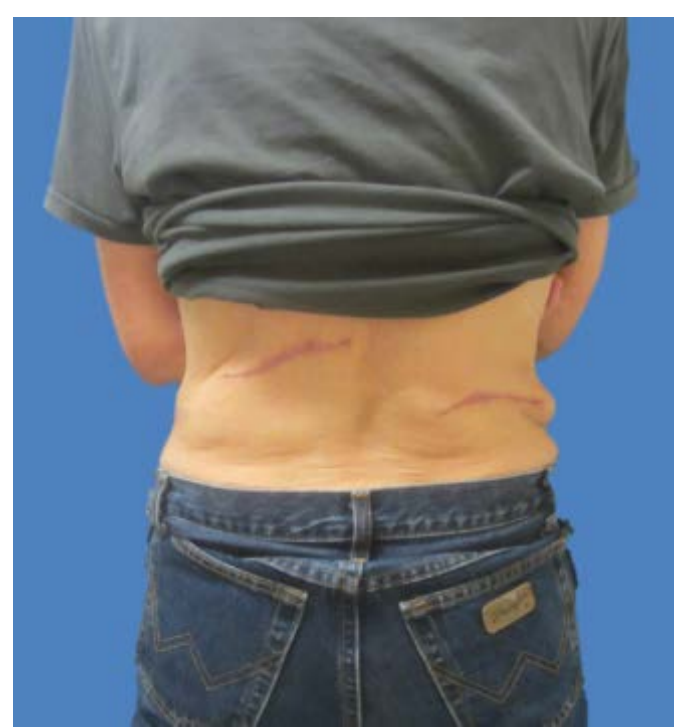

Figure 4: Postoperative view upon 6 months follow up with immature scars.

reports in the literature and slow progression with delayed diagnosis. The treatment of MSL is unsatisfactory as there is a high recurrence rate. Weight loss and alcohol consumption cessation have no effect 
on lipoma growth, while liposuction and surgical excision are often associated with recurrence [8]. Accurate diagnosis of MSL is important in order to evaluate the patients for the above mentioned systemic comorbidities. Furthermore, as $90 \%$ of MSL cases are related to high alcohol intake, alcohol abuse must be ruled out and if present, patients must be referred for appropriate care. Surgeons should bear in mind that clinical presentation may vary, as for our patient, and must be familiar with this disease and its management.

\section{References}

1. McTighe S, Chernev I (2014) Intramuscular lipoma: a review of the literature. Orthop Rev (Pavia) 6: 5618.

2. De La Cruz Monroy MF, Durani P, Offer GJ (2015) Unusual case of finger lipoma: a case report and literature review. J Plast Reconstr Aesthet Surg 68: 284-286.

3. Ardeleanu V, Chicos S, Georgics C, Tutunaru D (2013) Multiple Benign Symmetric Lipomatosis - A Differential Diagnosis of Obesity. Chirurgia 108: 580-583.

4. Tadisina KK, Mlynek KS, Hwang LK, Riazi H, Papay FA, et al. (2015) Syndromic lipomatosis of the head and neck: a review of the literature. Aesthetic Plast Surg 39: 440-448.

5. González-García R, Rodríguez-Campo FJ, Sastre-Pérez J, Muñoz-Guerra MF (2004) Benign Symmetric Lipomatosis (Madelung's Disease): Case Reports and Current Management. Aesth Plas Surg 28: 108-112.

6. Brea-García B, Cameselle-Teijeiro J, Couto-González I, Taboada-Suárez A González-Álvarez E (2013) Madelung's disease: comorbidities, fatty mass distribution, and response to treatment of 22 patients. Aesthetic Plast Surg 37 409-416.

7. Zielińska-Kaźmierska B, Lewicki M, Manowska B (2015) Madelung disease Postepy Dermatol Alergol 32: 400-403.

8. Mimica M, Pravdic D, Nakas-Icindic E, Karin M, Babic E, et al. (2013) Multiple symmetric lipomatosis: a diagnostic dilemma. Case Rep Med 836903.

9. Rabler F, Goetze S, Elsner P (2016) Abdominal variant of benign symmetric lipomatosis (Launois-Bensaude syndrome) imitating obesity. J Eur Acad Dermatol Venereol 30: 460-461.

10. Verhelle N, Nizet JL, Van den Hof B, Guelinckx P, Heymans O (2003) Liposuction in Benign Symmetric Lipomatosis: Sense or Senseless? Aesth Plast Surg 27: 319-321.

11. Sharma N, Hunter-Smith DJ, Rizzitelli A, Rozen WM (2015) A surgical view on the treatment of Madelung's disease. Clin Obes 5: 288-290. 